\title{
Alocação de Investigações Especiais E Comuns na Polícia Federal com o Uso da Teoria dos Jogos: Aplicação do Modelo Principal-Agente e do Valor de Shapley
}

\author{
ALLOCATION OF SPECLAL AND COMMON \\ InVESTIGATIONS IN the FEDERAL POLICE Using \\ Game Theory: ApPlication of the PRINCIPAL- \\ Agent Model and The Shapley Value
}

\begin{abstract}
ASIGNACIÓN DE INVESTIGACIONES ESPECLALES Y Comunes en la Policía Federal Medlante Teoría de Juegos: ApLiCACIÓN deL Modelo PRINCIPALAGENTE Y EL VALOR DE SHAPLEY
\end{abstract}

Submetido em 03 de janeiro de 2020.

Aceito em 02 de maio de 2021

Marco Aurélio Faveri

Polícia Federal, Recife/PE, Brasil

faveri.maf@pf.gov.br

(G) http://lattes.cnpq.br/2441760361396309

\begin{abstract}
RESUMO
O propósito deste trabalho é a construção, para a Polícia Federal, de um modelo de otimização e mecanismo de aplicação para a utilização de informações extraídas de policiais investigadores, que auxiliem decisores na distribuição de investigações. Foram apresentadas as metodologias principais de investigação policial atualmente empregadas pela organização, as quais costumam gerar dificuldades ao gestor no momento de identificar pessoal adequado a cada tipo de tarefa. Para solução, foram utilizadas abstrações racionais da Teoria dos Jogos, com destaque aos conceitos da teoria Principal-Agente e do valor de Shapley, tendo-se construído um modelo que associa ambas as técnicas. Inova o modelo na mescla de conceitos racionalizantes que orientam ao atingimento de pontos ótimos para a administração de equipes, sem a utilização de benefícios
\end{abstract}


pecuniários, de modo a se congregar qualidades e anseios do profissional em benefício da instituição e em detrimento de considerá-lo mero cumpridor de tarefas. Faz-se necessária a continuidade do estudo, com a aplicação contínua do modelo a casos concretos para aferição de possíveis melhorias e correções no modelo, bem como o desenvolvimento de metodologias para a avaliação de capacidades e preferências do Agente no que tange à familiaridade a investigaçóes especiais.

Palavras chave: gestão policial; teoria dos jogos; Principal-Agente; valor de Shapley; investigação.

\section{ABSTRACT}

The aim of this paper is to design an optimization model and application mechanism for the use by the Federal Police of information elicited from police investigators, to support Decision Makers in the distribution of investigations. The main police investigation methodologies currently used by the organization were presented, which usually generate difficulties for the management when identifying appropriate personnel for each type of task. For a solution, rational abstractions from Game Theory were used, with emphasis on the concepts of Principal-Agent theory and Shapley's value, having built a model that combines both techniques. The model innovates in the mix of rationalizing concepts that guide the achievement of optimal points for the management of teams, without the use of pecuniary benefits, in order to gather the professional's qualities and desires for the benefit of the institution, without considering him/her a mere fulfiller of tasks. It is necessary to continue the study, with the continuous application of the model to concrete cases to assess possible improvements and corrections in the model, as well as the development of methodologies for the assessment of the Agent's capabilities and preferences with regard to familiarity with special investigations.

KEYwORDS: police management; game theory; Principal-Agent; Shapley value; investigation.

\section{RESUMEN}

El propósito de este trabajo es la construcción para la Policía Federal de un modelo de optimización y mecanismo de aplicación para el uso de información extraída de los investigadores policiales, para asistir a los tomadores de decisiones en la distribución de las investigaciones. Se presentaron las principales metodologías de investigación policial que actualmente utiliza la organización, las cuales suelen generar dificultades al gestor a la hora de identificar al personal adecuado para cada tipo de tarea. Para la solución, se utilizaron conceptos de la Teoría de Juegos, con énfasis a la teoría Principal-Agente y al valor de Shapley, habiéndose construido un modelo que combina ambas técnicas. Innova el modelo en el mix de conceptos racionalizadores que orientan el logro de puntos óptimos para la gestión de equipos, sin beneficios pecuniarios, reuniendo las cualidades 
y deseos del profesional en beneficio de la institución y en detrimento de considerarlo un mero cumplidor de tareas. Es necesario continuar el estudio, con la aplicación continua del modelo a casos concretos para medición de posibles mejoras y correcciones en el modelo, así como el desarrollo de metodologías para la evaluación de las capacidades y preferencias del Agente en cuanto a familiaridad con investigaciones especiales.

Palabras Clave: gestión policial; teoría de juegos; Principal-Agente; valor de Shapley; investigación.

\section{INTRODUÇÃo}

A Polícia Federal é um órgão de segurança pública previsto na Constituição Federal, que exerce a função de polícia judiciária da União. Entre suas atividades está a apuração de infrações penais contra a ordem política e social ou em detrimento de bens, serviços e interesses da União (BRASIL, 2017b); tais como o combate ao terrorismo, à corrupção e aos crimes de colarinho branco, crimes contra o meio ambiente e lavagem de dinheiro, entre outros.

Estas investigações são realizadas seguindo-se a legislação penal e processual penal existente no país, interpretadas de acordo com as decisões do Poder Judiciário e instruídas através do inquérito policial presidido por um delegado de polícia (BRASIL, 2013), ao qual incumbe a definição e determinação das diligências policiais que precisam ser realizadas, respeitado o ordenamento jurídico vigente, para, assim, concluir-se pela existência ou não de um fato criminoso, apontando-se ou não indícios de autoria (BRASIL, 2017a).

A Polícia Federal está presente nas 27 Unidades da Federação brasileira, em suas capitais, bem como em outros 95 municípios (POLÍCIA FEDERAL, 2013). Composta por 13.053 servidores públicos, conforme dados de dezembro de 2020 (BRASIL, 2021), responsáveis pela execução das atividades meio e fim da organização, entre as quais a solução de inquéritos policiais, tendo, em junho de 2021, alcançado cerca de 85 mil inquéritos simultaneamente em andamento (POLÍCIA FEDERAL, 2021).

Como a maior parte das organizações, a estrutura da Polí- 
cia Federal é fundada no princípio da hierarquia (BRASIL, 1965), existindo um forte esquema organizacional (POLÍCIA FEDERAL, 2020) para definição de metas e prioridades, bem como para a execução dos trabalhos que lhe são afetos.

É a partir deste ponto que surgem os problemas a serem debatidos neste trabalho, um deles próprio de organizações estatais hierarquizadas, em que existem indivíduos em postos de comando superiores que representam a instituição perante à sociedade e recebem poderes públicos organizacionais direcionadores da atividade, mas que são executadas por servidores públicos de maneira delegada, na típica relação Principal-Agente.

O outro problema a ser enfrentado, mais uma vez decorrente da característica hierarquizada da organização em que os setores executivos se submetem às diretrizes e metas fixadas por unidades superiores, é o que diz respeito à divisão de tarefas, encargos e objetivos de otimização entre os agentes executores, não raro resultando em distribuição desproporcional e injusta de atividades.

Partindo-se destes problemas gerais, utilizando-se diretrizes racionais de teoria dos jogos, através deste trabalho criou-se um mecanismo de apoio à decisão e gerenciamento da investigação policial, norteador de pontos ótimos de distribuição de tarefas com a utilização da teoria Principal-Agente e do valor de Shapley, aplicáveis à realidade de uma delegacia de Polícia Federal especializada no combate e repressão a crimes de corrupção e desvio de recursos públicos, mas que poderá ser generalizada para outras unidades da Polícia Federal.

Este artigo está organizado em três partes principais: na Seção 2, apresenta-se a distribuição de trabalho na Polícia Federal; na Seção 3, expõe-se o referencial teórico que servirá de background à proposta de solução a ser apresentada na Seção 4, quando se construirá o modelo e o mecanismo de aplicação. Por fim, segue-se a conclusão do trabalho com a discussão dos resultados obtidos, suas limitações e a identificação de estudos que poderão ser futuramente desenvolvidos. 


\section{Problemas na Distribuição de Trabalho na Polícia Federal}

Entre as várias atividades desenvolvidas pela Polícia Federal, a que mais se destaca é a investigação de infrações penais de interesse da União, materializada no inquérito policial, que é um procedimento administrativo no qual são juntados os documentos que comprovam os fatos e os indícios de autoria, consistindo em todos os atos necessários à elucidação do caso, ao arbítrio do delegado de polícia, a ser utilizado para a prossecução penal perante o Poder Judiciário (PITO$\mathrm{MBO}, 2016)$.

Ocorre que ante a grande quantidade de fatos criminosos a serem elucidados, cada qual exigindo diferentes níveis de especialização e esforço investigativo e operacional, já há algum tempo a Polícia Federal se vale de conceitos de operaçôes especiais militares para o estabelecimento de equipes responsáveis por investigações consideradas prioritárias.

Sob a ótica militar, uma operação especial requer modos, táticas, técnicas, procedimentos e equipamentos específicos a serem empregados em ambientes hostis, negados ou sensíveis sob o aspecto político ou diplomático, caracterizados por sensitividade temporal, natureza clandestina ou encoberta, baixa visibilidade, trabalho com forças estrangeiras, necessidade de orientação regional e cultural, com alto grau de risco (USA, 2014) ;ou, de forma mais simplificada, uma operação especial é conduzida por forças especialmente treinadas, equipadas e apoiadas para um alvo específico, cuja destruição, eliminação ou resgate seja politicamente ou militarmente necessário (MCRAVEN, 1996).

A partir desse norte, no que concerne aos trabalhos da Polícia Federal em sua função de polícia judiciária da União, as investigações realizadas com o emprego de conceitos de operações especiais, aqui denominadas, também e simplesmente, de investigações especiais, são aquelas realizadas através de ações planejadas e concentradas, que utilizam recursos extraordinários e equipe que atua com prazo definido ao fim de neutralização do indivíduo ou grupo criminoso e obtenção de provas (SILVA, 2017). 
Assim, tais investigações especiais, que também são materializadas em um ou mais inquéritos policiais, representam uma quantidade muito pequena do total de investigações realizadas - totalizando apenas 536 deflagradas no ano de 2019 , ou seja, cerca $0,6 \%$ do total de inquéritos policiais em andamento -, mas demandam esforço muito maior de trabalho e apresentam resultados significativamente mais elevados no combate à criminalidade (BRASIL, 2020) na comparação unitária com inquéritos comuns.

Dessa forma, distinguindo-se as tarefas a serem realizadas entre investigações especiais e comuns, surge na organização o problema de distribuição de tais inquéritos entre o efetivo de delegados responsáveis por conduzi-las. Isso porque, conforme conceitos expostos, a investigação especial exige dedicação, treinamento e desenvolvimento focados no atingimento ao objetivo, algo que nem todos policiais estão aptos, desejam ou são vocacionados a enfrentar, mesmo porque não há contraprestação financeira diferenciada.

Sob outro ponto, a massa de investigações simultaneamente em andamento, sejam casos comuns ou especiais, também é objeto de gerenciamento, sobre a qual são estabelecidas metas a partir de indicadores mensuráveis, como tempo médio de desenvolvimento; quantidade por unidade policial; quantidade por delegado de polícia, etc., não sendo tarefa fácil ao decisor a individualização de tais objetivos.

Então é corriqueiro que órgãos superiores de gestão fixem metas gerais para redução de carga de inquéritos já antigos, por exemplo, distribuindo o objetivo de maneira linear entre todas as unidades ou servidores, o que pode representar uma solução não igualitária na repartição do trabalho a ser executado.

Tem-se assim uma melhor delimitação dos problemas a serem tratados neste artigo, quais sejam, a necessidade de otimização da distribuição e proporção entre investigações comuns e especiais, bem como a necessidade de repartição de metas gerenciais gerais de maneira igualitária, os quais serão agora tratados à luz da teoria dos jogos. 


\section{Background: Teoria Dos Jogos, Conceitos E LITERATURA}

A teoria dos jogos pode ser definida como o estudo de modelos matemáticos de conflito e cooperação entre decisores racionais, isto é, trata-se de uma teoria que proporciona técnicas matemáticas gerais para análise de situações em que dois ou mais indivíduos tomam decisões que influenciarão em outros (MYERSON, 1997).

Para tanto, tem-se como princípio que os indivíduos, ora jogadores, farão uma escolha racional, ou seja, tomarão a melhor decisão de acordo com suas preferências, entre todas as possíveis ações, e sem restrições qualitativas, de modo que sua racionalidade reside na consistência de suas decisões frente aos diferentes conjuntos de alternativas disponíveis, e não de acordo com a natureza de suas vontades ou aversões (OSBORNE, 2002).

Os modelos de teoria dos jogos são representações abstratas de situações da vida real, de forma que tal abstração permite que seja usada para o estudo de um amplo conjunto de fenômenos, por meio do uso da matemática, para expressar formalmente as ideias, facilitando a definição de conceitos de maneira precisa e dando consistência às ideias para se explorar as implicações e suposições (OSBORNE; RUBINSTEIN, 1994).

Não obstante isso, é através das aplicações práticas que se entende a teoria, já que argumentos formais sobre jogos abstratos ajudam, mas desempenham um papel menor, enquanto as aplicações ilustram o processo de construção do modelo, de modo que a variedade de aplicações mostra que questões semelhantes surgem em diferentes áreas e que as mesmas ferramentas podem ser aplicadas em cada ambiente (GIBBONS, 1992).

Em razão disso, pode-se encontrar na literatura atual exemplos de aplicações de teoria dos jogos nas mais diversas áreas, tais como cadeia de suprimentos (IVANOV; DOLGUI, 2020; ZHANG, Y.; HEZARKHANI, 2021); energia (CHENG; YU, 2019; CONG; WANG; JIANG, 2019; MORSTYN; TEYTELBOYM; MCCULL$\mathrm{OCH}, 2019$ ); engenharia de softwares e tecnologia (ASHRAF; 
NEWAGY; HAFEZ, 2019; GAVIDIA-CALDERON et al., 2020; MOSCATO; PICARIELLO; SPERLÍ, 2019); comportamento político, militar e econômico (COLBERT; KOTT; KNACHEL, 2020; DEANGELO; MCCANNON, 2020; HOLLYER; ROSENDORFF; VREELAND, 2019; TALEIZADEH; SADEGHI, 2019; WEI; CHAN, 2019); segurança pública (CASKEY; WASEK; FRANZ, 2018; GINGERICH; OLIVEROS, 2018; IMANPOUR et al., 2019); entre outros.

A teoria dos jogos compreende diversos modelos básicos, além de outros que possam ainda vir a ser deduzidos, cada qual adaptável da versão teórica à realidade cotidiana, cabendo ao estudioso o esforço de identificar se algum deles é profícuo ao esforço de solucionar um problema posto.

No caso deste trabalho, verificou-se que as racionalidades propostas na relação Principal-Agente e na teoria desenvolvida por Lloyd Shapley ( 1953) podem ser úteis para a solução de algumas demandas da Polícia Federal relacionadas à distribuição mais conveniente de trabalhos investigativos, conforme subseções seguintes.

\subsection{Modelo Principal-Agente ou Teoria da Agência}

Define-se a relação de agência como um contrato em que uma pessoa (Principal) engaja outra pessoa (Agente) a realizar algum serviço em seu nome, com a delegação de alguma autoridade decisória, de modo que, se ambas as partes maximizarem as suas utilidades, haja uma boa razão para acreditar que nem sempre o Agente irá agir no melhor interesse do Principal (JENSEN; MECKLING, 1976).

Assim, a estrutura básica do modelo é a dicotomia entre as duas partes, Principal e Agente, em que o primeiro delega ao segundo a execução de uma atividade com certo poder decisório, advindo daí uma relação assimétrica de conhecimentos entre um e outro.

A delegação pode ser motivada pela possibilidade de benefício obtido com a divisão de tarefas; pela falta de tempo, habilidade ou por qualquer outra forma de limitação do Principal, de modo que o Agente pode ter acesso a informações que não estão disponíveis ao Principal (LAFFONT; MARTIMORT, 2002). 
Da lacuna de informações é que advém os problemas nessa relação que se multiplica no cenário organizacional contemporâneo, eis que qualquer empreendimento público ou privado, com ou sem fins lucrativos, se pauta na divisão e distribuição de tarefas. Entretanto, os objetivos de cada ente participante dessa relação frequentemente são distintos e, muitas vezes, se contrapóem, o que pode causar sérios prejuízos à instituição.

Deste modo, a teoria da agência está preocupada em resolver dois problemas que podem ocorrer nessa relação Principal-Agente: o primeiro é quando existem desejos ou objetivos conflitantes e é difícil ou custoso ao Principal verificar o que o Agente está realmente fazendo, isto é, não se pode conferir se ele está se comportando adequadamente; o segundo é o risco de compartilhamento que surge quando Principal e Agente se portam de maneira diferente diante de um risco. Cabe então à teoria determinar o contrato mais eficiente a governar o relacionamento entre estas partes (EISENHARDT, 1989).

O custo de oportunidade da tarefa, a correta tecnologia usada e a habilidade em se usar essa tecnologia são exemplos de informações que podem se tornar de conhecimento privado do Agente, a chamada seleção adversa (adverse selection), assim como considera-se perigo moral (moral hazard), a perda da habilidade em controlar a performance do Agente, algo que não pode ser contratado, já que não há como se verificar o seu valor (LAFFONT; MARTIMORT, 2002).

No sentido inverso, o adequado tratamento dessa relação pode sim multiplicar os esforços e potencializar os objetivos de cada um, atenuando ou anulando as contrariedades, aplicando-se incentivos ou, ao menos, reduzindo-se as insatisfações.

Assim, o foco da teoria da agência é determinar contratos que eficientemente governem a relação Principal-Agente à luz dos problemas de adverse selection e moral hazard, o que é obtido geralmente por intermédio de contratos de comportamento orientado ou de resultado orientado, investimento em sistemas de informação que controlem o oportunismo, ou se tenta alinhar os interesses do agente por incentivos (WAGNER, 2019). 
Tradicionalmente, duas condições são necessárias para que o Agente se submeta a ordens do Principal: a) que aquele não se importe "muito" com qual tarefa este escolherá ou que seja compensado de alguma forma pela possibilidade deste escolher uma tarefa indesejada e; b) que seja vantajoso para este oferecer àquele uma compensação adicional se não puder prever, com certeza, qual tarefa será a mais adequada (SIMON, 1951), sendo que a função de satisfação $(S)$ para as partes pode ser descrita nas Equações 1 e 2.

$$
\begin{aligned}
& S_{p}=F_{p}(x)-q_{p} w \\
& S_{a}=F_{a}(x)+q_{a} w
\end{aligned}
$$

Em que $p$ e $a$ representam o Principal e o Agente, respectivamente, para $F(x)$ sendo o resultado da execução da tarefa, o incentivo $(q)$ e o salário $(w)$, do que se percebe ser razoável supor que $S \geq 0, F(x)$ $\geq 0$ e $q w>0$, já que seria irracional admitir um empreendimento em que se obtenha valores negativos de satisfação ou resultado, ainda que se admita serem nulos, assim como a remuneração deve ser superior a zero, já que se assume existir delegação remunerada de tarefa.

Por certo que a aceitação em se realizar a tarefa $(x)$ envolve não somente benefícios pecuniários, mas também a utilidade gerada por vários aspectos não remuneratórios, como a atratividade com a equipe, o nível de disciplina exigida, as relações pessoais como amizade e respeito entre a equipe ou mesmo um bom computador para trabalhar (JENSEN; MECKLING, 1976).

Não obstante isso, as tratativas remuneratórias pressupõem, por parte do Principal, uma expectativa do resultado da tarefa, sem que tenha certeza antecipadamente se se concretizará o desfecho da maneira desejada, residindo aí sua desvantagem quanto à assimetria de informações.

Para dirimir esse problema no que se refere à seleção adversa (adverse selection), ou seja, uma eventual má escolha quanto à capacidade de o Agente realizar a tarefa, o Principal deve seguir duas etapas quando da contratação (LAFFONT; MARTIMORT, 2002). Primeiro descrever um conjunto de resultados que poderão ser alcançados a despeito da falta de informações, apontando-se também um conjunto de restrições de compatibilidade de incentivos onde houver informa- 
ção assimétrica, aliado a um conjunto de restrições de participação, criando-se assim um contrato de incentivos possíveis. Depois, deve-se proceder a uma análise normativa e otimizar a função objetivo do Principal dentro do conjunto de resultados viáveis de incentivos.

Já quanto à lacuna de informações a respeito de eventuais ações que o Agente possa escolher praticar quando da execução da tarefa (moral hazard), as quais possam resultar em alteração do valor do negócio, tais como o modo de dirigir de um motorista ou a dedicação de um tratador de animais, ou ainda o modo de conduzir uma investigação policial, o conflito está nas ações escolhidas para a realização da tarefa contratada.

Em um contexto de moral hazard, o resultado vinculado ao esforço do agente é uma questão de sorte. Entretanto, o principal pode ajustar um contrato baseado na performance observável do agente. Assim, o contrato deve induzir, a um custo razoável, um alto esforço do Agente a despeito da impossibilidade de diretamente condicionar a recompensa a sua ação, de forma que um primeiro passo é identificar incentivos que induzem a um esforço positivo, satisfazendo assim as restrições de incentivo. Além disso, induzir a participação voluntária do Agente cria um padrão de restrições de participação, resultando no contrato de incentivos possíveis. A partir daí, o Principal dá preferência àquele que implemente o nível positivo de esforço ao custo mínimo (LAFFONT; MARTIMORT, 2002).

A respeito do tema, Eisenhardt (1989) propõe que para contratos baseados em resultado o Agente tende a se comportar de acordo com os interesses do Principal e que, quando este tem as informações para se verificar o comportamento daquele, o Agente mais uma vez tende a agir de acordo com os interesses do Principal.

Além disso, identificou que sistemas de informação, incerteza de resultados, aversão ao risco do Agente, a programabilidade das tarefas e a duração do relacionamento de agência são positivamente relacionados a contratos baseados em comportamento e negativamente relacionados a contratos de resultado. Enquanto que a aversão ao risco do Principal, o conflito de objetivos entre um e outro e a mensurabilidade de resultados são positivamente relacionados a contratos de resultado e negativamente a contratos de comportamento (EISENHARDT, 1989). 
Como será melhor detalhado em seção específica, na aplicação proposta neste trabalho haverá um misto entre contrato de resultado e contrato de comportamento, a partir dos tipos de tarefas a serem distribuídas, assim como, haja vista limitações no gerenciamento de incentivos remuneratórios, eventuais benefícios serão contratados a partir de satisfações na forma, tipo e ambiente de trabalho.

A literatura atual é farta em trabalhos de aplicação da teoria da agência, conforme se pode notar nas seguintes áreas: recursos hídricos (DARBANDSARI et al., 2020); gestão de recursos humanos (AN, W.; KWEON, 2017; BARRACHINA; GONZÁLEZ-CHORDÁ, 2020; ZHANG, C. et al., 2020); inspeção de fronteiras (BAKSHI; GANS, 2010); contratos (HU; CHEN; WANG, 2018; KERKKAMP; VAN DEN HEUVEL; WAGELMANS, 2019); comércio de carbono (CAI; SINGHAM, 2018); tecnologia (ZHANG, R.; ZHU, 2020); comportamento policial (GOTTSCHALK, 2018); entre outros.

\subsection{Valor De Shapley}

Entre os fundamentos da teoria dos jogos está a hipótese de que os jogadores podem avaliar, em suas escalas de utilidade, cada cenário que surgir como resultado de um jogo. Foi a partir deste enunciado que Lloyd Shapley (1953) incluiu na classe de cenários a probabilidade de se jogar o jogo, ou seja, aferir-se qual a efetiva participação do jogador no jogo, isso se ele participar.

Pautou-se, para tanto, em pressupostos do seminal trabalho de Von Neumann e Morgenstern (1944) os quais defendem que a utilidade é objetiva e transferível, que os jogos envolvem interesses cooperativos e que, nestas condições, estão adequadamente representados por suas funções características.

Assim, o valor de Shapley de um jogo é definido com referência a outros jogos possíveis, isto é, uma função que atribui um único perfil de payoff viável a cada jogo de coalizão, em que um perfil de $p a$ yoff é viável se a soma de seus componentes for $v(N)$, que é considerado o perfil de eficiência, dado que $\{N, v\}$ sejam uma coalizão de payoff transferível (OSBORNE; RUBINSTEIN, 1994). 
Os procedimentos de barganha que consideram jogos não cooperativos e cooperativos entre duas pessoas foram bem explorados por John Nash (1950, 1951, 1953), advindo a formulação de Shapley para jogos cooperativos de $n$-pessoas, que considera coalisóes, isto é, qualquer subconjunto não vazio de um conjunto de jogadores, tratando-se então de uma construção axiomática em que o autor levanta quais tipos de propriedades se pode esperar para uma solução satisfatória a se alcançar a melhor alocação de payoff esperada em uma coalisão (MYERSON, 1997).

Assim sendo, dado que o jogador $i$ é um dummy em $v$ se $\Delta_{i}(S)$ $=\mathrm{v}(\{i\})$ para toda coalisão $S$ que exclui $i$, e que os jogadores $i$ e $j$ são intercambiáveis em $v$ se $\Delta_{i}(S)=\Delta_{j}(S)$ para toda coalisão $S$ que não contenham nem $i$ e nem $j$, o autor definiu os seguintes axiomas e teorema (GUL, 1989; MYERSON, 1997; OSBORNE; RUBINSTEIN, 1994; SHAPLEY, 1953):

- Simetria: se $i$ e $j$ são intercambiáveis em $v$, então $\phi_{i}(v)=\phi_{j}(v), \mathrm{o}$ que afirma que o valor é essencialmente a propriedade de um jogo abstrato.

- Eficiência: estabelece que o jogador dummy (i) é o que não estará no jogo e não contribui para a coalisão vencedora, então em $v$ ele será $\phi_{i}(v)=v(\{i\}), \operatorname{com} v(\{i\})=0$, enquanto a coalisão carrier $N$ de $v$ é a que reúne os jogadores que dividirão o resultado, sem qualquer alocação ao dummy, de modo que $\sum_{N} \phi_{i}(v)=v(N)$.

- Agregação ou aditividade: em que para quaisquer dois jogos $v \mathrm{e}$ $w$ tem-se $\phi(v+w)=\phi(v)+\phi(w)$, ou seja, quando dois jogos independentes forem combinados, seus valores podem ser adicionados jogador por jogador

O teorema resultante, cuja função valor satisfaz os axiomas enunciados para jogos com finitas coalisões carriers $N$, é dado pela Equação 3, para todo universo de jogadores $(i \in U)$.

$$
\phi_{i}(v)=\sum_{s \subseteq N}\left[\frac{(s-1) !(n-s) !}{n !}\right] \cdot[v(S)-v(S-i)]
$$

Diante disso, o valor de Shapley é uma ferramenta poderosa para avaliar a força estrutural em um jogo de coalisão (MYERSON, 
1997). Isso porque, a conjunção de seus axiomas permite afirmar que a ordem de entrada do jogador na coalisão não é fator decisivo para a divisão dos valores, os quais serão equitativamente divididos de acordo com a participação de cada jogador, levando-se em consideração todas as possibilidades de coalisão.

O valor resultante da fórmula de Shapley concebe uma solução cuja motivação é normativa, o que descreve uma razoável ou justa forma de dividir ganhos (ou perdas) em uma cooperação, consideradas as realidades estratégicas capturadas pela forma característica (MASCOLELL; WHINSTON; GREEN, 1995).

O valor de Shapley é então definido como a soma das contribuições dos $i$ 's em todas as possíveis coalisões $S$, na média de todas as ordens possíveis de formação de coalizão, podendo-se dizer que o jogador que recebe como recompensa o valor de Shapley está recebendo sua contribuição líquida (de utilidade), em respeito ao princípio da contribuição, ou seja, concede ao jogador um payoff de utilidade ponderado pelas necessidades recíprocas igual à sua contribuição média de utilidade ponderada pelas necessidades recíprocas para os outros jogadores, um ótimo de Pareto (BROCK, 1979).

Desde sua concepção o valor de Shapley vem sendo amplamente utilizado como método mais justo para distribuição de resultados, cabendo mencionar alguns trabalhos recentes na biologia (SUN et al., 2020; WICKE; FISCHER, 2019), na distribuição de lucros e fundos (AN, Q. et al., 2019; CUBUKCU, 2020; DEMETRESCU et al., 2019; TENG et al., 2019), no meio ambiente (KELLNER; SCHNEIDERBAUER, 2019; STELLINGWERF et al., 2019), na distribuição de água (LI; ZHAO; GOVINDARAJU, 2019), em transporte público (GNECCO; HADAS; SANGUINETI, 2020), na alocação de patrulhas policiais (WU et al., 2020), entre outros.

\section{Construção do Modelo e Proposta de AplicaÇão}

Para a solução do problema submetido à análise, qual seja, a melhor distribuição de operações especiais e investigações comuns 
entre os delegados disponíveis em uma delegacia de Polícia Federal, é possível utilizar-se a racionalidade proporcionada pelo modelo Principal-Agente, apoiada por uma alocação estável de trabalho desenhada a partir dos conceitos formulados por Shapley.

Assim, nesta seção serão estruturadas as funções de utilidade que se aproximam da melhor representação do modelo de agência existente na Polícia Federal, para distribuição do trabalho investigativo, considerando o delegado-chefe de uma unidade específica na função de Principal, pois gerencia os recursos humanos e materiais locais e presta contas à alta gestão do Poder Público e à sociedade, destinatária última do serviço; tendo na função de Agentes os demais delegados lotados naquela unidade, que lideram equipes de investigação, têm o conhecimento do trabalho e realizam junto com os demais policiais o esforço necessário à realização da atividade policial.

$\mathrm{Na}$ sequência, será estruturado um mecanismo para alocação das operações especiais e das investigações comuns a partir do valor de Shapley, proporcionando também ao gestor o desenvolvimento de estratégias que possibilitem a redução ou incremento de atributos relacionados às investigações, comumente utilizados como mensuradores de produtividade, com a adequada distribuição de metas.

Ao fim, será testado o modelo a partir de dados simulados não reais, mas representativos de uma Delegacia de Polícia Federal especializada no combate e repressão a crimes de corrupção e desvios de recursos públicos federais, que poderá ser generalizado para utilização em unidades de quaisquer municípios ou de outras especializações investigativas.

\subsection{Máximo Esforço Operacional Policial}

A Polícia Federal brasileira exerce, entre outras funções estabelecidas na Constituição Federal (BRASIL, 2017b), a atividade de investigação de crimes cometidos em detrimento de bens e interesses públicos federais, fazendo parte da Administração Pública direta do Poder Executivo Federal.

Em razão disso, por intermédio do Ministério da Justiça e Se- 
gurança Pública - MJSP, ao qual está vinculada, a Polícia Federal conta com orçamento para realização de suas atividades, incluindo o que é disponibilizado ao pagamento de remunerações ao efetivo, bem como verbas de diárias devidas por conta do deslocamento temporário de servidores entre circunscrições, sendo estes os valores monetários que poderiam ser considerados como custos ao trabalho realizado pelos policiais.

Conforme destacado na seção anterior, o modelo Principal- $A$ gente estimula a concessão de incentivos em acréscimo à remuneração do empregado, algo que deverá ser considerado com reservas neste trabalho, já que o policial federal recebe apenas remuneração fixa na forma de subsídio (BRASIL, 2016), sendo que, muito embora as diárias de deslocamento possam constituir uma espécie de parcela variável, destina-se ao pagamento das despesas de hospedagem, alimentação e transporte do servidor em outra circunscrição (BRASIL, 1990, 2006).

Não obstante isso, como já mencionado, a aceitação e estímulo quanto à tarefa a ser realizada não ocorre apenas a partir de benefícios monetários, mas também de outros fatores a serem estimulados, já que como a proposta desta pesquisa é o apoio ao trabalho do administrador local, este não controla a remuneração pecuniária, cabendo-lhe tão somente o gerenciamento do esforço operacional e do ambiente de trabalho.

Assim, na função utilidade do Principal (Equação 4), tem-se como variáveis o orçamento para pagamento da remuneração do servidor (b), algo que não está sob seu controle, e o equivalente ao esforço operacional sob a visão do gestor, considerado como cumprimento do dever policial $(d)$, este sim sob gerenciamento direto do administrador local, de modo que a Administração Pública e a sociedade recebem o fruto do dever policial $(d)$ ao custo do orçamento $(b)$.

$$
A=d-b
$$

Por seu turno, o delegado investigador, no papel de Agente nesta modelagem, tem a receber pelo seu trabalho a remuneração em forma de subsídio $(r)$, eventuais pagamentos de diárias de deslocamento $(q)$, ao custo de empregar o esforço pessoal operacional (e), conforme Equação 5. 


$$
B=r+q-e
$$

Assumindo-se que todo o custo financeiro é empregado no pagamento de remuneração e diárias, bem como que o esforço operacional do Agente reverte-se no cumprimento do dever policial, é possível alcançar a Equação 6.

$$
d-b=r+q-e \Rightarrow e-(r+q)=r+q-e
$$

Como já mencionado, não será objeto deste trabalho o tratamento do orçamento policial ou da remuneração financeira devida ao servidor, mas somente do esforço operacional, com o gerenciamento da força humana de trabalho sob o aspecto da distribuição de funções, contexto em que tal esforço pode ser representado na Equação 7.

$$
e=\left(\frac{\delta+\frac{\varphi}{n} k}{2}\right)^{2}
$$

Para tanto, convenciona-se denominar:

$\delta$, o correspondente à taxa de satisfação operacional $(\omega)$, multiplicada pela quantidade de operações especiais $(g)$ a ser desenvolvida pelo delegado $(\delta=\omega \cdot g)$, sendo que o índice de satisfação poderá ser construído a partir de critério único, programação ou por um modelo de Multiple Criteria Decision Making/Aiding (MCDM/A), com informações obtidas diretamente do delegado investigador, reduzindo-se a assimetria de informações própria do modelo Principal-Agente, a medir assim o nível de familiaridade ou aversão do profissional com o tipo de trabalho (investigações especiais). A construção desse critério único ou modelo MCDM/A, por si só, poderá constituir pesquisa específica, de maneira que neste trabalho será considerada uma taxa de satisfação 1 por ocasião da aplicação.

$\varphi$, a taxa de inquéritos policiais a ser obtida também por critério único, modelo $\mathrm{MCDM} / \mathrm{A}$ ou técnicas de otimização, poderá corresponder a um somatório de importância de inquéritos, de antiguidade, de adequação correcional ou qualquer outra forma de medição de produtividade ou evento da natureza. 
$n$, a quantidade de inquéritos distribuída ao delegado investigador pelo Principal.

$k$, a constante para adequação entre a quantidade de inquéritos e de operações existentes, a partir da modelagem obtida com o uso do valor de Shapley para as variáveis.

A partir desses parâmetros, uma vez conhecida a percepção do investigador a respeito das exigências maiores de uma operação especial $(\omega)$ e descartando-se os atributos financeiros $(b, r$ e $q)$, cabe ao Principal promover a otimização do esforço operacional (e), conforme descrito na Equação 8:

$\operatorname{Max}_{\epsilon} B=\left(\frac{\delta+\frac{\varphi}{n} k}{2}\right)^{2}=\frac{(\delta n+k \varphi)^{2}}{4 n^{2}}=\left(\frac{\delta^{2}}{4}+\frac{\delta k \varphi}{2 n}+\frac{k^{2} \varphi^{2}}{4 n^{2}}\right)$

$\mathrm{Na}$ sequência, encontram-se as derivadas parciais e condições de primeira ordem para $n$ e $\delta$ (Equações 9 e 10):

$$
\begin{gathered}
f^{\prime}(n)=\frac{k \varphi(\delta n+k \varphi)}{2 n^{3}} \Rightarrow \frac{k \varphi(\delta n+k \varphi)}{2 n^{3}}=0 \Rightarrow n=-\frac{k \varphi}{\delta} \\
f^{\prime}(\delta)=\frac{\delta n+k \varphi}{2 n} \Rightarrow \frac{\delta n+k \varphi}{2 n}=0 \Rightarrow \delta=-\frac{k \varphi}{n}
\end{gathered}
$$

Por certo que, conforme é perceptível, a maximização do esforço em investigações especiais implica em manter em zero a quantidade de inquéritos comuns e vice versa. Mas por várias razões de ordem prática, de especialização de conhecimento investigativo e mesmo porque uma investigação especial se materializa em um ou mais inquéritos comuns, o delegado que se dedica ao trabalho especial, também conduz inquéritos. Em razão disso, a constante $k$ precisa ser um valor negativo para que se mantenha o equilíbrio na maximização da alocação de trabalhos, o que resulta no gráfico da função de otimização (Figura 1), para $k=-0,8$ e $\varphi=100$. 


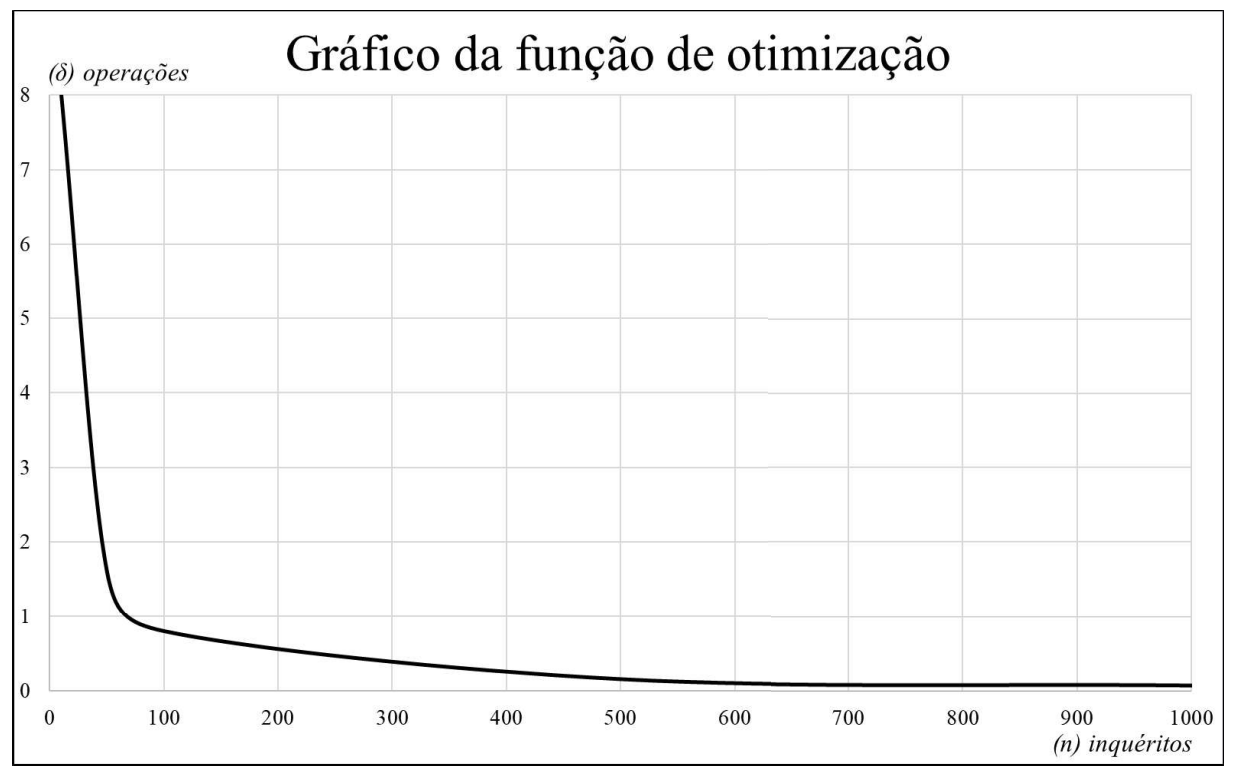

Figura 1 - gráfico da função de otimização: índice de operações vs inquéritos

\subsection{Aplicação do Modelo em uma Delegacia}

Uma vez encontrada a função que maximiza a relação entre inquéritos comuns e investigações especiais, com o auxílio do valor de Shapley será demonstrada a aplicação do modelo em uma delegacia especializada no combate e repressão a crimes de corrupção e desvio de recursos públicos, mas que poderá ser generalizada para outras unidades policiais.

Aqui será considerada uma delegacia com cinco delegados $\{\mathrm{A}, \mathrm{B}, \mathrm{C}, \mathrm{D}$ e $\mathrm{E}\}$ que atuam em investigações comuns e especiais; uma quantidade atual de 214 inquéritos policiais e de 13 operações especiais a serem desenvolvidas por estes mesmos servidores. Como estratégias de gestão, pretende-se que em um período qualquer seja a carga de inquéritos reduzida para $80 \%$ do montante atual e que a antiguidade média dos inquéritos em andamento, hoje de 662 dias, seja reduzida para $70 \%$ no mesmo período, conforme Tabela 1 . Os valores serão arredondados para o número inteiro superior se $\geq 0,5$.

Tabela 1 - Quantitativos atuais de inquéritos e resultados com valor de Shapley

\begin{tabular}{cccccccc} 
Delegado & $\mathrm{A}$ & $\mathrm{B}$ & $\mathrm{C}$ & $\mathrm{D}$ & $\mathrm{E}$ & Total & Média \\
\hline Inquéritos atuais & 14 & 68 & 41 & 36 & 55 & 214 & 43 \\
\hline $\begin{array}{c}\text { Antiguidade média } \\
\text { atual }\end{array}$ & 513 & 503 & 953 & 663 & 680 & 3311 & 662 \\
\hline
\end{tabular}




\begin{tabular}{cccccccc}
\hline Inquéritos após Shapley & 11 & 58 & 31 & 27 & 45 & 171 & 34 \\
\hline $\begin{array}{c}\text { Antiguidade após } \\
\text { Shapley }\end{array}$ & 351 & 344 & 682 & 464 & 477 & 2318 & 464 \\
\hline
\end{tabular}

Diante dessas informações, é possível estabelecer-se dois cenários de alocação dos trabalhos, o primeiro focado na distribuição de operações especiais, dada a quantidade de inquéritos comuns e antiguidade esperados por delegado no fim do período; e o segundo focado na distribuição de inquéritos atuais a partir do número de operações já alocadas.

Cenário 1: conforme Tabela 2, dá-se a distribuição de operações especiais $(\delta)$ a partir dos inquéritos $(n)$ e antiguidade $(\varphi)$ esperados no final do período. Deixou-se de arredondar o resultado até o número inteiro para que o gestor possa melhor interpretar os dados e decidir finalmente a alocação.

Tabela 2 - alocação de operações policiais

\begin{tabular}{cccccccc}
\hline Delegado & A & B & C & D & E & Total & Média \\
\hline $\boldsymbol{n}$ & 11 & 58 & 31 & 27 & 45 & 171 & 34 \\
\hline $\boldsymbol{k}$ & $-0,144$ & $-0,144$ & $-0,144$ & $-0,144$ & $-0,144$ & - & - \\
\hline $\boldsymbol{\phi}$ & 351 & 344 & 682 & 464 & 477 & 2318 & 464 \\
\hline $\boldsymbol{\delta}$ & $\mathbf{4 , 8 0}$ & $\mathbf{0 , 8 8}$ & $\mathbf{3 , 2 4}$ & $\mathbf{2 , 5 1}$ & $\mathbf{1 , 5 7}$ & $\mathbf{1 3}$ & - \\
\hline
\end{tabular}

Cenário 2: tem-se agora um processo em dois momentos. A partir da alocação das operaçóes $(\delta)$, são distribuídos os inquéritos atuais $(n)$, considerando a antiguidade média total atual $(\varphi)$. Então aplica-se o valor de Shapley para se atingir o objetivo de redução da carga $\left(n^{*}\right)$ e da antiguidade esperada $\left(\varphi^{*}\right)$ tomando-se, pois, a nova distribuição de antiguidade média $\left(\varphi^{\prime}\right)$ decorrente da alocação $(n)$ dos inquéritos. Note-se que com a distribuição aqui proposta, alterou-se a antiguidade média inicial de cada delegado, que somadas chegavam a 3.311 dias, mas passou para 3.505 dias, de modo que $70 \%$ corresponde a 2.453 dias.

Tabela 3 - alocação de inquéritos policiais

\begin{tabular}{cccccccc}
\hline Delegado & A & B & C & D & E & Total & Média \\
\hline $\boldsymbol{n}$ & 17 & 85 & 28 & 42 & 42 & 214 & 43 \\
\hline $\boldsymbol{k}$ & $-0,128$ & $-0,128$ & $-0,128$ & $-0,128$ & $-0,128$ & - & - \\
\hline $\boldsymbol{\varphi}$ & 662 & 662 & 662 & 662 & 662 & 3311 & 662 \\
\hline
\end{tabular}




\begin{tabular}{cccccccc}
\hline$\delta$ & 5 & 1 & 3 & 2 & 2 & 13 & - \\
\hline$\varphi^{\prime}$ & 670 & 551 & 889 & 741 & 654 & 3505 & 701 \\
\hline$n^{*}$ & $\mathbf{1 3}$ & $\mathbf{7 4}$ & $\mathbf{2 1}$ & $\mathbf{3 2}$ & $\mathbf{3 2}$ & $\mathbf{1 7 1}$ & $\mathbf{3 4}$ \\
\hline$\varphi^{*}$ & $\mathbf{4 6 7}$ & $\mathbf{3 7 8}$ & $\mathbf{6 3 2}$ & $\mathbf{5 2 1}$ & $\mathbf{4 5 6}$ & $\mathbf{2 4 5 3}$ & $\mathbf{4 9 1}$ \\
\hline
\end{tabular}

No Cenário 2, ainda é possível uma terceira etapa para reajuste das operações face a distribuição dos inquéritos obtida após estabelecidas as metas de redução $\left(n^{*} \mathrm{e} \varphi^{*}\right)$, algo que, no exemplo sob análise poderá resultar em um valor inteiro a maior ou a menor, haja vista as frações resultantes, não se substituindo assim mais uma vez a decisão final do gestor.

\subsection{Discussão dos Resultados}

No modelo proposto, seguindo a aplicação demonstrativa que poderá ser ajustada para situações concretas, foram impostas duas estratégias gerenciais para redução de índices de acompanhamento, quais sejam, a quantidade bruta de inquéritos policiais e a redução do tempo médio de tramitação das investigações.

O quantitativo de procedimentos está no núcleo do estudo, mas poderia ter sido utilizado sem o estabelecimento de meta, assim como no lugar da meta de redução do tempo médio, poderia ter sido utilizada qualquer outra medida valorativa do trabalho a ser realizado, acompanhada ou não de uma proposta a ser atingida.

Há que se recordar que este trabalho apresenta duas propostas que poderão ser utilizadas separadamente ou em conjunto. Uma concernente à alocação de recursos e tarefas a partir do estabelecimento de objetivos a serem alcançados, com a utilização do valor de Shapley. Outra proposta, à luz da teoria da agência, no intuito de conseguir estabelecer um contrato adequado entre o administrador da unidade policial (Principal) e o delegado que conduzirá a investigação (Agente), isso a partir da identificação de suas preferências quanto ao trabalho especial, que serão aproveitadas no cálculo.

Do mesmo modo, ainda que não tenha sido utilizado na aplicação, uma medida de importância dos inquéritos (FAVERI, 2019) pode ser útil também como ajuste no momento do estabelecimento 
do contrato Principal-Agente, a servir até mesmo como benefício em contrapartida a investigações especiais desgastantes.

Nisso reside a inovação do modelo a ser utilizado em ambiente investigativo policial local, generalizável a outras unidades ou mesmo a outro tipo de organização, já que mescla conceitos racionalizantes, que se direcionam ao atingimento de pontos ótimos para a administração de equipes, seja sob o aspecto de valorização meritocrática, seja como meio de extrair as melhores qualidades do profissional em benefício da instituição, ou ainda de atentar-se a inclinações subjetivas, deixando de considerar o Agente como mero cumpridor de tarefas, ainda que sem empregar benefícios pecuniários como incentivos, lidando outrossim com a noção de deveres e preferências.

O modelo contribui, em um ambiente de acordo entre toda a equipe, para um ajuste mais personalizado possível de contrato quanto aos tipos de trabalho a serem balanceados, quais sejam, operações especiais e investigações comuns. Isso porque as primeiras se adequam mais a contratos de resultado, eis que por definição demandam maior investimento de recursos e dedicação do Agente, voltados a um objetivo específico.

De outra ponta, as investigações comuns ocorrem em volume muito superior e precisam necessariamente compartilhar recursos para potencializar a produção, exigindo-se menos resultados concretos, os quais passam a ser encarados admitindo-se perdas estatisticamente controladas, impondo-se ações e comportamentos "corretos" do Agente.

Nesse sentido, o modelo ajuda a identificar Agentes com características pessoais que se adequam mais ou menos a um ou outro tipo de trabalho, utilizando essa informação elicitada para incentivar o policial a atuar em atividade que detenha mais aptidão ou interesse, algo que também é vantajoso ao Principal (Administração Pública), reduzindo o quanto possível a assimetria de informações entre as partes envolvidas nessa relação de trabalho. 


\section{Conclusão}

Utilizando os conceitos de racionalidade da Teoria dos Jogos, em especial a teoria Principal-Agente e o valor de Shapley, o objetivo deste artigo foi a construção de um mecanismo de otimização que auxiliasse o decisor na resolução de dois problemas enfrentados pela Polícia Federal, quais sejam, a redução da assimetria de informações entre os administradores e os delegados investigadores, bem como a distribuição mais justa da carga de trabalho a ser executada.

Diante disso, foram apresentados os tipos de trabalho realizados pela organização e que foram objeto de estudo, ou seja, as operações especiais que são aquelas com propósitos específicos e que concentram recursos, treinamento e apoio direcionados, exigindo dedicação maior por parte do investigador; e as investigações comuns, que ocorrem em volume bem superior e que compartilham o restante dos recursos disponíveis.

Assim, sempre é difícil ao decisor identificar policiais com perfis adequados a tal e qual atividade a ser executada, bem como balancear a distribuição das investigações de maneira equilibrada, justa e que atenda às expectativas, tanto da instituição quanto dos servidores públicos envolvidos, em um cenário de escassa ou nula manobra recompensatória pecuniária.

Os conceitos da teoria da agência e do valor de Shapley foram apresentados com o objetivo de identificar soluções possíveis aos problemas da organização policial especificados, acompanhados da literatura clássica de construção das doutrinas, bem como de aplicações recentes nas mais diversas áreas econômicas, políticas e sociais.

A partir disso, foi construído modelo, tendo como finalidade a obtenção do máximo esforço operacional policial em que, sem se socorrer de incentivos financeiros, buscou-se identificar preferências do Agente quanto ao trabalho mais desgastante que, para alguns, é mais recompensador, cotejando essa informação com inquéritos comuns, propondo distribuições mais justas inclusive para o atingimento de objetivos institucionais. 
A aplicação do modelo com dados simulados, mas realísticos de uma delegacia especializada, demonstra a robustez do modelo, estando apto a ser empregado em caráter experimental em unidades reais de produção, com o fim de aprimoramento e posteriores proposições de melhorias.

Novos trabalhos se mostram adequados e necessários para melhor elicitação das preferências do Agente no que concerne à familiarização ao trabalho em operações especiais, inclusive detalhando-se neste tipo de trabalho eventuais inclinações entre temáticas específicas, como o combate ao desvio de recursos públicos, a crimes contra o meio ambiente, ao tráfico de drogas, ao terrorismo, entre outros, sempre com o intuito de reduzir a assimetria de informações para com o Principal, buscando identificar a vocação da própria unidade policial a partir de seus policiais.

BIOGRAFIA DO AUTOR:

\section{Marco Aurélio Faveri}

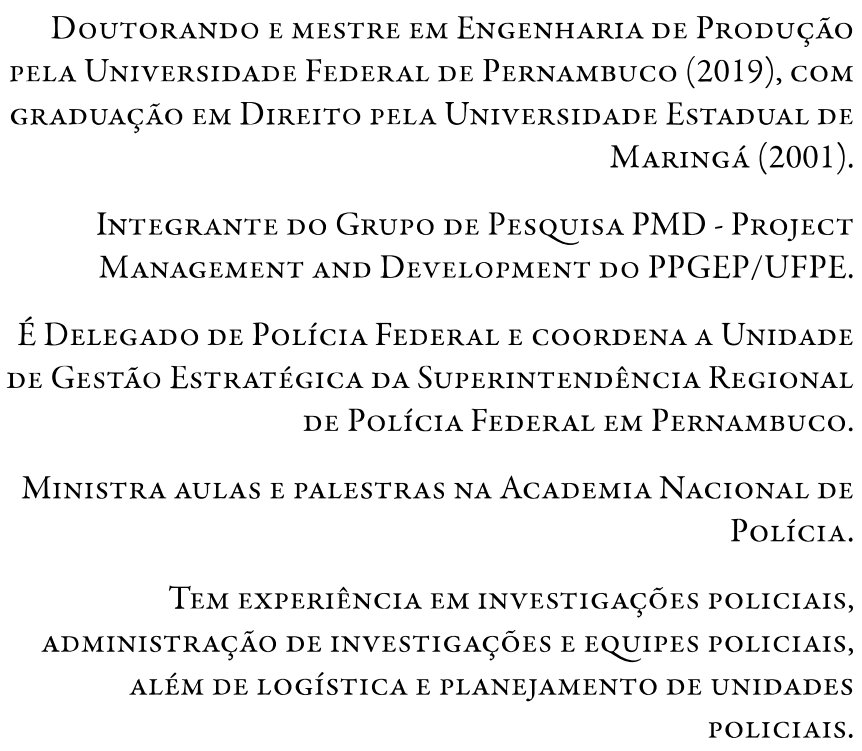

\section{REFERÊNCIAS}

AN, Qingxian et al. Resource sharing and payoff allocation in a three-stage system: Integrating network DEA with the Shapley value method. Omega, [s. l.], v. 85, p. 16-25, 2019. Disponível em: https:// 
doi.org/10.1016/j.omega.2018.05.008

AN, Weihua; KWEON, Yesola. Do higher government wages induce less corruption? Cross-country panel evidence. Journal of Policy Modeling, [s. l.], v. 39, n. 5, p. 809-826, 2017. Disponível em: https://doi.org/10.1016/j.jpolmod.2017.03.001

ASHRAF, Yara; NEWAGY, Fatma; HAFEZ, Ismail. Performance evaluation of cooperative and non-cooperative MIMO cognitive radio networks. IET Communications, [s. l.], v. 13, n. 20, p. 3588-3594, 2019. Disponível em: https://doi.org/10.1049/ietcom.2019.0522

BAKSHI, Nitin; GANS, Noah. Securing the Containerized Supply Chain: Analysis of Government Incentives for Private Investment. Management Science, [s. l.], v. 56, n. 2, p. 219-233, 2010. Disponível em: https://doi.org/10.1287/mnsc.1090.1105

BARRACHINA, Alex; GONZÁLEZ-CHORDÁ, Víctor M. Error reporting and the performance of nursing management: a gametheoretic study. IMA Journal of Management Mathematics, [s. l.], v. 31, n. 4, p. 445-468, 2020. Disponível em: https://doi.org/10.1093/ imaman/dpz019

BRASIL. Código de Processo Penal: Decreto-Lei no 3.689/1941. $1^{\mathrm{a} e d .}$ Brasília/DF: Senado Federal, Coordenação de Edições Técnicas, 2017a. E-book.

BRASIL. Constituição da República Federativa do Brasil de 1988: texto constitucional promulgado em 5 de outubro de 1988, com as alterações determinadas pelas Emendas Constitucionais de Revisão $n^{\circ}$ 1 a 6/94, pelas Emendas Constitucionais no 1/92 a 99/2017. Brasília/ DF: Senado Federal, Coordenação de Edições Técnicas, 2017b. E-book.

BRASIL. Decreto ${ }^{\circ}$ 5.992, de 19 de dezembro de 2006, que dispõe sobre a concessão de diárias no âmbido da administração federal direta, autárquica e fundacional, e dá outras providências. Brasília/ DF, 2006. Disponível em: http://www.planalto.gov.br/ccivil_03/_ ato2004-2006/2006/decreto/d5992.htm

BRASIL. Lei no 12.830, de 20 de junho de 2013, que dispõe sobre a investigação criminal conduzida pelo Delegado de Polícia. Brasília/ 
DF, 2013. Disponível em: http://www.planalto.gov.br/ccivil_03/_ ato2011-2014/2013/lei/112830.htm

BRASIL. Lei n ${ }^{\circ} 13.371$, de 14 de dezembro de 2016, que altera a remuneração de serviodres públicos. Brasília/DF, 2016. Disponível em: http://www.planalto.gov.br/ccivil_03/_ato2015-2018/2016/ lei/L13371.htm

BRASIL. Lei n ${ }^{\circ} 4.878 / 1965$, de 3 de dezembro de 1965 , que dispõe sobre o regime jurídico peculiar dos funcionários policiais civis da União e do Distrito Federal. [s. l.], 1965. Disponível em: http:// www.planalto.gov.br/ccivil_03/leis/L4878compilado.htm

BRASIL. Lei $n^{\circ} 8.112$, de 11 de dezembro de 1990, que dispõe sobre o regime jurídico dos servidores públicos civis da União, das autarquias e das fundações públicas federais. Brasília/DF, 1990. Disponível em: http://www.planalto.gov.br/ccivil_03/leis/ 18112 cons.htm

BRASIL. Painel estatistico de pessoal. [S. l.], 2021. Disponível em: https://www.painel.pep.planejamento.gov.br/. Acesso em: $10 \mathrm{fev}$. 2020.

BRASIL. Relatório de gestão 2019 do Ministério da Justiça e Segurança Pública. Brasília/DF: [s.n.], 2020. Disponível em: https://www. gov.br/mj/pt-br/acesso-a-informacao/auditorias/auditoria-a-partirde-2019/rrmjsp2019.

BROCK, Horace W. A game theoretic account of social justice. Theory and Decision, [s. l.], v. 11, n. 3, p. 239-265, 1979. Disponível em: https://doi.org/10.1007/BF00126380

CAI, W.; SINGHAM, D.I. A principal-agent problem with heterogeneous demand distributions for a carbon capture and storage system. European Journal of Operational Research, [s. l.], v. 264, n. 1, p. 239-256, 2018. Disponível em: https://doi.org/10.1016/j. ejor.2017.06.009

CASKEY, Trevor R.; WASEK, James S.; FRANZ, Anna Y. Deter and protect: crime modeling with multi-agent learning. Complex \& Intelligent Systems, [s. l.], v. 4, n. 3, p. 155-169, 2018. Disponível em: https://doi.org/10.1007/s40747-017-0062-8 
CHENG, Lefeng; YU, Tao. Game-Theoretic Approaches Applied to Transactions in the Open and Ever-Growing Electricity Markets From the Perspective of Power Demand Response: An Overview. IEEE Access, [s. l.], v. 7, p. 25727-25762, 2019. Disponível em: https://doi.org/10.1109/ACCESS.2019.2900356

COLBERT, Edward JM; KOTT, Alexander; KNACHEL, Lawrence $P$. The game-theoretic model and experimental investigation of cyber wargaming. The Journal of Defense Modeling and Simulation: Applications, Methodology, Technology, [s. l.], v. 17, n. 1, p. 21-38, 2020. Disponível em: https://doi.org/10.1177/1548512918795061

CONG, Hao; WANG, Xu; JIANG, Chuanwen. Robust coalitional game theoretic optimisation for cooperative energy hubs with correlated wind power. IET Renewable Power Generation, [s. l.], v. 13, n. 13, p. 2391-2399, 2019. Disponível em: https://doi.org/10.1049/ iet-rpg.2018.6232

CUBUKCU, K. Mert. The problem of fair division of surplus development rights in redevelopment of urban areas: Can the Shapley value help? Land Use Policy, [s. l.], v. 91, p. 104320, 2020. Disponível em: https://doi.org/10.1016/j.landusepol.2019.104320

DARBANDSARI, Pedram et al. An agent-based conflict resolution model for urban water resources management. Sustainable Cities and Society, [s. l.], v. 57, p. 102112, 2020. Disponível em: https://doi. org/10.1016/j.scs.2020.102112

DEANGELO, Gregory; MCCANNON, Bryan C. Psychological game theory in public choice. Public Choice, [s. l.], v. 182, n. 1-2, p. 159-180, 2020. Disponível em: https://doi.org/10.1007/s11127019-00676-6

DEMETRESCU, Camil et al. On the Shapley value and its application to the Italian VQR research assessment exercise. Journal of Informetrics, [s. l.], v. 13, n. 1, p. 87-104, 2019. Disponível em: https://doi.org/10.1016/j.joi.2018.11.008

EISENHARDT, Kathleen M. Agency Theory: An Assessment and Review. Academy of Management Review, [s. l.], v. 14, n. 1, p. 57-74, 1989. Disponível em: https://doi.org/10.5465/amr.1989.4279003

FAVERI, Marco Aurelio. Análise multicritério de investigaçôes 
policiais: avaliação em momento de escassa informação e tratamento de imprecisóes. 145 f. 2019. - Universidade Federal de Pernambuco, [s. l.], 2019. Disponível em: https://repositorio.ufpe.br/ handle/123456789/35397

GAVIDIA-CALDERON, Carlos et al. Game-theoretic analysis of development practices: Challenges and opportunities. Journal of Systems and Software, [s. l.], v. 159, p. 110424, 2020. Disponível em: https://doi.org/10.1016/j.jss.2019.110424

GIBBONS, Robert. Game theory for applied economist. New Jersey: Princeton University Press, 1992.

GINGERICH, Daniel W.; OLIVEROS, Virginia. Police Violence and the Underreporting of Crime. Economics \& Politics, [s. l.], v. 30, n. 1, p. 78-105, 2018. Disponível em: https://doi.org/10.1111/ ecpo. 12102

GNECCO, Giorgio; HADAS, Yuval; SANGUINETI, Marcello. Public transport transfers assessment via transferable utility games and Shapley value approximation. Transportmetrica A: Transport Science, [s. l.], p. 1-26, 2020. Disponível em: https://doi.org/10.108 $0 / 23249935.2020 .1799112$

GOTTSCHALK, Petter. Opportunistic behavior in the principalagent model of policing. International Journal of Police Science \& Management, [s. l.], v. 20, n. 2, p. 109-115, 2018. Disponível em: https://doi.org/10.1177/1461355718763453

GUL, Faruk. Bargaining Foundations of Shapely Value.

Econometrica, [s. l.], v. 57, n. 1, p. 81, 1989. Disponível em: https:// doi.org/10.2307/1912573

HOLLYER, James R.; ROSENDORFF, B. Peter; VREELAND, James Raymond. Transparency, Protest and Democratic Stability. British Journal of Political Science, [s. l.], v. 49, n. 4, p. 1251-1277, 2019. Disponível em: https://doi.org/10.1017/ S0007123417000308

HU, Duni; CHEN, Shou; WANG, Hailong. Robust reinsurance contracts with uncertainty about jump risk. European Journal of Operational Research, [s. l.], v. 266, n. 3, p. 1175-1188, 2018. Disponível em: https://doi.org/10.1016/j.ejor.2017.10.061 
IMANPOUR, Maryam et al. A microeconomic foundation for optimal money laundering policies. International Review of Law and Economics, [s. l.], v. 60, p. 105856, 2019. Disponível em: https://doi. org/10.1016/j.irle.2019.105856

IVANOV, Dmitry; DOLGUI, Alexandre. Viability of intertwined supply networks: extending the supply chain resilience angles towards survivability. A position paper motivated by COVID-19 outbreak. International Journal of Production Research, [s. l.], v. 58, n. 10, p. 2904-2915, 2020. Disponível em: https://doi.org/10.1080/0 0207543.2020 .1750727

JENSEN, Michael C.; MECKLING, William H. Theory of the firm: Managerial behavior, agency costs and ownership structure. Journal of Financial Economics, [s. l.], v. 3, n. 4, p. 305-360, 1976. Disponível em: https://doi.org/10.1016/0304-405X(76)90026-X

KELLNER, Florian; SCHNEIDERBAUER, Miriam. Further insights into the allocation of greenhouse gas emissions to shipments in road freight transportation: The pollution routing game. European Journal of Operational Research, [s. l.], v. 278, n. 1, p. 296-313, 2019. Disponível em: https://doi.org/10.1016/j.ejor.2019.04.007

KERKKAMP, R.B.O.; VAN DEN HEUVEL, W.; WAGELMANS, A.P.M. Robust pooling for contracting models with asymmetric information. European Journal of Operational Research, [s. l.], v. 273, n. 3, p. 1036-1051, 2019. Disponível em: https://doi.org/10.1016/j. ejor.2018.08.041

LAFFONT, Jean-Jacques; MARTIMORT, David. The Theory of Incentives. New Jersey: Princeton University Press, 2002. Disponível em: https://doi.org/10.2307/j.ctv7h0rwr

LI, Dongnan; ZHAO, Jianshi; GOVINDARAJU, Rao S.

Water benefits sharing under transboundary cooperation in the Lancang-Mekong River Basin. Journal of Hydrology, [s. l.], v. 577, p. 123989, 2019. Disponível em: https://doi.org/10.1016/j. jhydrol.2019.123989

MAS-COLELL, Andreu; WHINSTON, Michael Dennis; GREEN, Jerry R. Microeconomic Theory. New York: Oxford University Press, 1995. 
MCRAVEN, William H. Spec ops: case studies in special operations warfare theory \& practice. New York: Presidio Press, 1996.

MORSTYN, Thomas; TEYTELBOYM, Alexander; MCCULLOCH, Malcolm D. Bilateral Contract Networks for Peer-to-Peer Energy Trading. IEEE Transactions on Smart Grid, [s.l.], v. 10, n. 2, p. 2026-2035, 2019. Disponível em: https://doi. org/10.1109/TSG.2017.2786668

MOSCATO, Vincenzo; PICARIELLO, Antonio; SPERLÍ, Giancarlo. Community detection based on Game Theory. Engineering Applications of Artificial Intelligence, [s. l.], v. 85, p. 773-782, 2019. Disponível em: https://doi.org/10.1016/j. engappai.2019.08.003

MYERSON, Roger B. Game theory: analysis of conflict. Cambridge/ MA: Harvard University Press, 1997.

NASH, John. Non-Cooperative Games. The Annals of Mathematics, [s. l.], v. 54, n. 2, p. 286, 1951. Disponível em: https://doi. org/10.2307/1969529

NASH, John. The Bargaining Problem. Econometrica, [s. l.], v. 18, n. 2, p. 155, 1950. Disponível em: https://doi.org/10.2307/1907266

NASH, John. Two-Person Cooperative Games. Econometrica, [s. l.], v. 21, n. 1, p. 128, 1953. Disponível em: https://doi. org/10.2307/1906951

OSBORNE, Martin John. An introduction to game theory. New York/NY: Oxford University Press, 2002.

OSBORNE, Martin John; RUBINSTEIN, Ariel. A course in game theory. Cambridge/MA: MIT Press, 1994.

PITOMBO, Antônio Sérgio Altieri de Moraes. Criminal Law and Procedure. In: DEFFENTI, Fabiano; BARRAL, Welber (org.). Introduction to Brazilian Law. 2. ed. Alphen aan den Rijn: [s.n.], 2016. E-book.

POLÍCIA FEDERAL. Informações obtidas via Lei de Acesso à Informação no processo 08198.018320/2021-09. Brasília/DF: [s.n.], 2021.

POLÍCIA FEDERAL. Organograma da Policia Federal 2020. 
[S. l.], 2020. Disponível em: https://www.gov.br/pf/pt-br/ acesso-a-informacao/institucional/estrutura/@@download/file/ ORGANOGRAMA_2020.pdf. Acesso em: 10 fev. 2021. POLÍCIA FEDERAL. Portaria $n^{\circ}$ 3.997/2013-DG/PF, de 24 de outubro de 2013: Define as circunscrições oficiais das Superintendências Regionais e das Delegacias de Policia Federal Descentralizadas. Brasília/DF: [s.n.], 2013. Disponível em: https://www.gov.br/pf/ pt-br/acesso-a-informacao/institucional/portaria-3997-2013-dg-pf. pdf

SHAPLEY, Lloyd S. Value for n-Person Games. In: KUHN, H W; TUCKER, A W (org.). Contribution to the Theory of Games. Annals of Mathematics Studies. New Jersey: Princeton University Press, 1953. p. 307-317.

SILVA, Élzio Vicente da. Operaçôes especiais de polícia judiciária: e ruptura de planos de ataque terrorista. Barueri/SP: Novo Século, 2017.

SIMON, Herbert A. A Formal Theory of the Employment Relationship. Econometrica, [s. l.], v. 19, n. 3, p. 293, 1951. Disponível em: https://doi.org/10.2307/1906815

STELLINGWERF, H.M. et al. Fair gain allocation in eco-efficient vendor-managed inventory cooperation. Journal of Cleaner Production, [s.l.], v. 231, p. 746-755, 2019. Disponível em: https:// doi.org/10.1016/j.jclepro.2019.05.232

SUN, Min Woo et al. Game theoretic centrality: a novel approach to prioritize disease candidate genes by combining biological networks with the Shapley value. BMC Bioinformatics, [s.l.], v. 21, n. 1, p. 356, 2020. Disponível em: https://doi.org/10.1186/s12859-020-03693-1

TALEIZADEH, Ata Allah; SADEGHI, Razieh. Pricing strategies in the competitive reverse supply chains with traditional and e-channels: A game theoretic approach. International Journal of Production Economics, [s. l.], v. 215, p. 48-60, 2019. Disponível em: https://doi.org/10.1016/j.ijpe.2018.06.011

TENG, Yue et al. Using cooperative game theory to determine profit distribution in IPD projects. International Journal of Construction Management, [s. l.], v. 19, n. 1, p. 32-45, 2019. Disponível em: 
https://doi.org/10.1080/15623599.2017.1358075

USA. Special Operations: Joint Publication 3-05. Washington/DC: [s. n.], 2014.

VON NEUMANN, John; MORGENSTERN, Oskar. Theory of Games and Economic Behavior. Princeton: Princeton University Press, 1944. Disponível em: https://doi.org/10.2307/j.cttl r2gkx WAGNER, Dirk Nicolas. The Opportunistic Principal. Kyklos, [s. l.], v. 72, n. 4, p. 637-657, 2019. Disponível em: https://doi. org/10.1111/kykl.12213

WEI, Fang; CHAN, Wang. The Cooperative Stability Evolutionary Game Analysis of the Military-Civilian Collaborative Innovation for China's Satellite Industry. Mathematical Problems in Engineering, [s. l.], v. 2019, p. 1-17, 2019. Disponível em: https://doi. org/10.1155/2019/3938716

WICKE, Kristina; FISCHER, Mareike. On the Shapley Value of Unrooted Phylogenetic Trees. Bulletin of Mathematical Biology, [s. l.], v. 81, n. 2, p. 618-638, 2019. Disponível em: https://doi. org/10.1007/s1 1538-018-0392-8

WU, Cheng-Kuang et al. A Game Theory Approach for Assessment of Risk and Deployment of Police Patrols in Response to Criminal Activity in San Francisco. Risk Analysis, [s. l.], v. 40, n. 3, p. 534-549, 2020. Disponível em: https://doi.org/10.1111/risa.13411

ZHANG, Chang et al. The Influence of Demographic Characteristics on Employee Promotion: Research Based on Data Mining and Game Theory. Wireless Communications and Mobile Computing, [s. l.], v. 2020, p. 1-12, 2020. Disponível em: https://doi. org/10.1155/2020/8814733

ZHANG, Rui; ZHU, Quanyan. FlipIn: A Game-Theoretic Cyber Insurance Framework for Incentive-Compatible Cyber Risk Management of Internet of Things. IEEE Transactions on Information Forensics and Security, [s. l.], v. 15, p. 2026-2041, 2020. Disponível em: https://doi.org/10.1109/TIFS.2019.2955891 ZHANG, Yumeng; HEZARKHANI, Behzad. Competition in dual-channel supply chains: The manufacturers' channel selection. European Journal of Operational Research, [s. l.], v. 291, n. 1, 
p. 244-262, 2021. Disponível em: https://doi.org/10.1016/j. ejor.2020.09.031 


\section{INFORMAÇÕES ADICIONAIS E DECLARAÇÕES DOS AUTORES}

$$
\text { (integridade cientifica) }
$$

Declaração de conflito de interesse: $\mathrm{O}(\mathrm{s})$ autor(es) confirma $(\mathrm{m})$ não haver conflitos de interesse na condução desta pesquisa e na redação deste artigo.

Declaração de autoria: Todos e apenas os pesquisadores que atendem os requisitos de autoria deste artigo são listados como autores; todos os coautores são integralmente responsáveis por este trabalho em sua totalidade.

Declaração de originalidade: $\mathrm{O}(\mathrm{s})$ autor(es) assegura(m) que o texto aqui publicado não foi previamente divulgado em qualquer outro local e que a futura republicação apenas será feita com expressa referência desta publicação original; também atesta $(\mathrm{m})$ que não há plágio de material de terceiros ou autoplágio.

\section{Como Citar (Abnt BrasiL)}

FAVERI, Marco Aurélio. Alocação de investigações especiais e comuns na Polícia Federal com o uso da Teoria dos Jogos: aplicação do modelo Principal-Agente e do valor de Shapley. Revista Brasileira de Ciências Policiais, Brasília, vol. 13, n. 7, p. 303336, jan./abr. 2022.

https://doi.org.br/10.31412/rbcp.v13i7.864

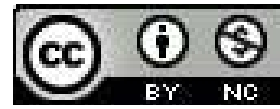

Esta obra está licenciada Com uma Licença Creative Commons Atribuição-NãoComercial 4.0 Internacional. 